\title{
Analysis of Anisotropic Pore Structures using Terahertz Spectroscopy and Imaging
}

\author{
Daniel Markl*, Cathy Ridgway ${ }^{\dagger}$, Prince Bawuah ${ }^{\ddagger}$, Patrick Gane ${ }^{\dagger \S}$, \\ Jarkko Ketolainen ${ }^{\top}$, Kai-Erik Peiponen ${ }^{\ddagger}$ and J Axel Zeitler* \\ * Department of Chemical Engineering and Biotechnology, University of Cambridge, UK \\ Email:dm733@cam.ac.uk \\ ${ }^{\dagger}$ Omya International AG, Oftringen, Switzerland \\ $\ddagger$ Institute of Photonics, University of Eastern Finland, P.O. Box 111, FI-80101 Joensuu, Finland \\ $\S$ School of Chemical Engineering, Department of Bioproducts and Biosystems, Aalto University, Aalto, Finland \\ ISchool of Pharmacy, Promis Centre, University of Eastern Finland, P.O. Box 1617, FI-70211, Kuopio, Finland
}

\begin{abstract}
This study demonstrates the analysis of anisotropic pore structures of highly porous pharmaceutical powder compacts by combining terahertz time-domain spectroscopy and in-situ measurements of the liquid penetration using terahertz pulsed imaging.
\end{abstract}

\section{INTRODUCTION AND BACKGROUND}

$\mathbf{T}$ HE pore structure plays an important role in many different materials as it often controls fluid flow through a material (e.g. geological formations or pharmaceutical tablets). In order to gain insight into the fluid flow through porous systems the pore structure must be measured and quantitatively described. A very promising methodology to measure pore properties is terahertz time-domain spectroscopy (THz-TDS) coupled with effective medium theory. The linear polarisation of the terahertz radiation can be utilised to study the predominant orientation and shape of pores by using an anisotropic effective medium approximation. The liquid flow through porous materials can be analysed by terahertz pulsed imaging (TPI), which is is capable of capturing liquid penetration kinetics that take place on timescales of seconds [1].

This study demonstrates the use of THz-TDS and TPI to quantitatively describe anisotropic pore structures and to analyse the liquid flow through the porous systems. We analysed 5 sets of pharmaceutical tablets consisting of functionalised calcium carbonate $\left(\mathrm{FCC}\right.$, Omyapharm ${ }^{\circledR}$, Omya International AG, Oftringen, Switzerland) with a nominal porosity, $f_{n}$, ranging from $45 \%$ to $65 \%$ with $5 \%$ increments.

\section{REsults}

The samples were measured by THz-TDS to determine the porosity and their predominant shape using the anisotropic Bruggeman model. This analysis yielded a sphericity of the pores of 1.7 and that the spheroidal shaped pores have a preferred orientation perpendicular to the compaction direction (see centre schematic in Figure 1b). We analysed the arrangement of pores on the basis of a structural $(S)$ parameter (Figure 1a and $\mathrm{b}$ ):

$$
S=\frac{1}{n_{U}^{2}-n_{L}^{2}}\left(\frac{n_{U}^{2} n_{L}^{2}}{n_{\mathrm{eff}}^{2}}-n_{L}^{2}\right)
$$

$n_{\text {eff }}$ is the effective refractive index of the powder compact measured by THz-TDS and $n_{L}$ and $n_{U}$ are the lower and upper Wiener bounds, respectively. The $S$ parameter is correlated with the oscillation frequency determined from in-situ TPI measurements of the liquid penetration. The anisotropic pore structure thus causes fluctuations of the liquid penetrating the powder compact.

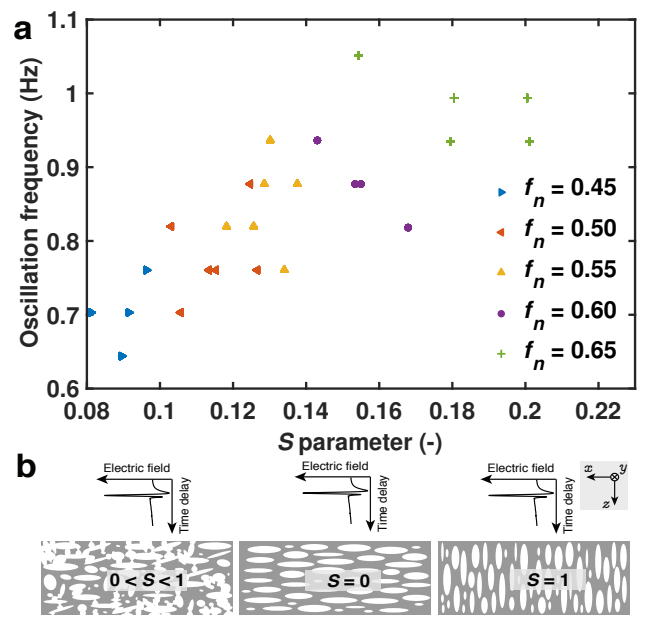

Fig. 1. Analysis of TPI and THz-TDS measurements. (a) The high frequency oscillations from the in-situ measurements of the liquid penetration by TPI as a function of the $S$ parameter of each sample. The $S$ parameter is determined for each sample by THz-TDS using Eq. 1. (b) Schematics of a tablet with different arrangements of the pores, which is captured by the $S$ parameter. $f$ is the porosity determined by THz-TDS.

\section{CONCLUSION}

This study demonstrates the application of THz-TDS and TPI to gain information about anisotropic pore structures and its impact on the liquid ingress into highly porous pharmaceutical tablets.

\section{REFERENCES}

[1] S. Yassin, D. J. Goodwin, A. Anderson, J. Sibik, D. Ian Wilson, L. F. Gladden, and J. A. Zeitler, "The Disintegration Process in Microcrystalline Cellulose Based Tablets, Part 1: Influence of Temperature, Porosity and Superdisintegrants," Journal of Pharmaceutical Sciences, vol. 104, no. 10 , pp. $3440-3450$, Oct. 2015. 